\title{
A CROSS-CULTURAL SIMULATION-BASED COMPARISON OF PRENATAL COUNSELING: AMERICAN AND DUTCH NEONATOLOGISTS' PERSPECTIVES
}

\author{
R. Geurtzen ${ }^{1}$, M. Hogeveen ${ }^{1}$, A. Rajani ${ }^{2}$, R. Chitkara ${ }^{2}$, T.A. Antonius ${ }^{1}$, J. Draaisma ${ }^{3}$, L.P. Halamek ${ }^{2}$ \\ ${ }^{1}$ Paediatrics and Neonatology, Radboud University Nijmegen, Medical Centre, Nijmegen, The Netherlands, \\ ${ }^{2}$ Centre for Advanced Paediatric and Perinatal Education, Lucile Packard Children's Hospital, Stanford \\ University, Stanford, CA, USA, ${ }^{3}$ Paediatrics, Radboud University Nijmegen, Medical Centre, Nijmegen, The \\ Netherlands
}

Background: Prenatal counseling at the threshold of viability is difficult and care guidelines differ worldwide. In the United States palliative comfort care is commonly advised below 23 completed weeks of gestation and an individualized approach is advised at $23-24$ weeks of gestation. At the time of this trial, in the Netherlands comfort care was recommended for any newborn under 25 completed weeks of gestation.

Objective: In this study we sought to determine similarities and differences in content of actual prenatal counseling at the limits of viability between American and Dutch neonatologists.

Subjects and methods: We compared counseling practice between 11 American and 11 Dutch neonatologists, using a simulation-based investigative methodology. Information was collected on the offered options for treatment and the discussed consequences of prematurity.

Results: Dutch neonatologists were more likely to explicitly advise a specific course of treatment whereas many of the American neonatologists simply provided several treatment options. However, when a specific form of treatment was advised, Americans recommended more aggressive care than their Dutch colleagues. American subjects offered comfort care as an option more frequently than the Dutch subjects. The groups exhibited few differences in the consequences of prematurity that they discussed with the simulated pregnant patients.

Conclusion: Dutch subjects were more directive in their approach than their American counterparts, offering fewer options for care and advocating for less aggressive interventions. American subjects were more likely to offer a wider range of therapeutic options without providing a recommendation for any specific option. 